\title{
Why are average faces attractive? The effect of view and averageness on the attractiveness of female faces
}

\author{
TIM VALENTINE, STEPHEN DARLING, and MARY DONNELLY \\ Goldsmiths College, University of London, London, England
}

\begin{abstract}
Images of faces manipulated to make their shapes closer to the average are perceived as more attractive. The influences of symmetry and averageness are often confounded in studies based on full-face views of faces. Two experiments are reported that compared the effect of manipulating the averageness of female faces in profile and full-face views. Use of a profile view allows a face to be "morphed" toward an average shape without creating an image that becomes more symmetrical. Faces morphed toward the average were perceived as more attractive in both views, but the effect was significantly stronger for full-face views. Both full-face and profile views morphed away from the average shape were perceived as less attractive. It is concluded that the effect of averageness is independent of any effect of symmetry on the perceived attractiveness of female faces.
\end{abstract}

A. L. Austin observed, in a letter written to Charles Darwin in 1877, that a composite of two faces was strikingly attractive. Mr. Austin placed two different portraits in a stereoscope and noticed that they fused into a single portrait. He wrote: "The faces blend in the most remarkable manner, producing in case of some ladies portraits, in every instance, a decided improvement in beauty" (Galton, 1878, p. 137). Psychologists have been intrigued by this phenomenon, which has been replicated in more formal studies (e.g., Langlois \& Roggman, 1990; Langlois, Roggman, \& Musselman, 1994; Rhodes \& Tremewan, 1996). The effect is found in male as well as female faces (Langlois \& Roggman, 1990) and across ethnic backgrounds (e.g., Perrett, May, \& Yoshikawa, 1994; Rhodes, Yoshikawa et al., 2001).

Identification of the determinants of facial attractiveness has become a central issue in evolutionary psychology. Attractiveness may signal some biological propensity for survival (i.e., "good genes") and preferences may serve to select an optimal mate. Symmetry may indicate stress-free growth, while averageness may indicate genetic diversity that boosts immunity to disease. Such preferences may serve also to maintain stability in the population. For reviews of the mechanisms of human facial attractiveness, see Kalick, Zebrowitz, Langlois, and Johnson (1998) and Thornhill and Gangestad (1999).

Alternatively, preferences in facial attractiveness could be an artifact of a general cognitive mechanism, but nevertheless be propagated through the population (the "perceptual bias" account). Distinctive faces are recognized more

Correspondence concerning this article should be addressed to T. Valentine, Department of Psychology, Goldsmiths College, University of London, New Cross, London SE14 6NW, England (e-mail: t.valentine@gold.ac.uk). easily than are typical faces, but typical faces are classified as a face, rather than a scrambled face, more quickly than distinctive faces (Valentine, 1991). These effects have been interpreted as evidence that faces are represented in a multidimensional face-space (Valentine, 1991, 2001). Further support for this framework comes from the observation that caricatured faces can be recognized more accurately than veridical faces (e.g., Lee \& Perrett, 2000). Facial distinctiveness (a converse measure of averageness) is negatively correlated with attractiveness (e.g., Rhodes, Sumich, \& Byatt, 1999). Such results suggest that there may be a role of general cognitive processing mechanisms that mediate perceived attractiveness of average faces.

Two accounts for the attractiveness of composite faces can be identified. ${ }^{1}$ (1) Composite faces are attractive because they are average in shape (the "averageness hypothesis"; e.g., Langlois \& Roggman, 1990). (2) Composite faces are attractive because they are symmetrical (the "symmetry hypothesis"; e.g., Rhodes, Roberts, \& Simmons, 1999). These hypotheses are not, of course, mutually exclusive. A third possibility is that composite faces are attractive because they have a smoothed skin texture. However, averageness is attractive even in line drawings of faces that do not include any skin texture (Rhodes \& Tremewan, 1996).

There have been a number of attempts to differentiate between the roles of the averageness and symmetry in the perception of attractiveness. The role of symmetry has been extensively researched. Symmetrical faces can be produced by combining half of a face split by a vertical midline with its mirror image. Such faces are perfectly symmetrical but are not perceived to be more attractive than the original faces (e.g., Kowner, 1996; Langlois et al., 1994; Samuels, Butterworth, Roberts, Graupner, $\&$ Hole, 1994). Therefore, it can be concluded that sym- 
metry is not a sufficient condition for a face to be attractive. However, this approach has been criticized on the grounds that symmetrical chimeric faces often look unnatural and so may not provide an appropriate test of the symmetry hypothesis (e.g., Rhodes, Roberts, \& Simmons, 1999; Rhodes, Sumich, \& Byatt, 1999).

Rhodes and her colleagues (Rhodes, Profitt, Grady, \& Sumich, 1998; Rhodes, Roberts, \& Simmons, 1999; Rhodes, Sumich, \& Byatt, 1999) have advocated a procedure in which faces are morphed (blended) with their mirror image to produce natural-looking faces in which the level of symmetry can be controlled. Blending a face with its mirror image in equal proportion produces an image that is perfectly symmetrical but is not average in shape. It was concluded that symmetry per se does affect the attractiveness of a face (Rhodes et al., 1998; Rhodes, Roberts, \& Simmons, 1999). Similarly, Perrett et al. (1999) used a technique in which the original image (texture) of each face was mapped onto the shape of a face created by averaging the position of landmarks from the two sides of a face. Thus, the face shape but not the texture was symmetrical. Perrett et al. (1999) found a preference for symmetrical faces.

Neither blending a face with its mirror image nor averaging face shape about the midline entirely disentangles the effects of symmetry and averageness. Such a blend will produce a more symmetrical face, but it will also be closer to the average (symmetrical) face shape by virtue of its symmetry. The resulting image will tend to have more average values on facial dimensions of variation than the original image because it is a blend of two asymmetrical images. Therefore, a confounding role of averageness could have contributed to effects attributed to symmetry.

The studies cited above do not address the question of whether average faces are attractive only because of their symmetry. Rhodes, Sumich, and Byatt (1999) used multiple regression to investigate whether ratings of symmetry and averageness predict perceived attractiveness. Both factors had independent effects. The experiments reported here used a novel approach to seek converging evidence for an independent effect of averageness on the perception of attractiveness, in the absence of any influence of symmetry. The simple expedient of using profile views of faces was employed. A profile view of a face does not have any axes of symmetry. Therefore, profile faces can be morphed toward or away from the average shape without introducing a confound with symmetry. Participants made a twoalternative forced choice between faces in which they were asked to select the most attractive face. A condition that used full-face views (in which the effects of symmetry and averageness were confounded) was included as a baseline and to allow comparison with previous studies.

\section{EXPERIMENT 1}

\section{Method}

Participants. There were 48 student participants ( 28 female, 20 male). Their age ranged from 18 to 41 years $(M=24$ years, 4 months; $S D=6$ years, 10 months).
Design. A $2 \times 3$ factorial repeated measures design was used. The two factors were view of face (full-face and profile) and the manipulation of the comparison face (three levels). The stimuli included four versions of each face: the veridical face (henceforth denoted $0 \%$ ), a $25 \%$ morph of the veridical face toward the average face $(-25 \%)$, a $50 \%$ morph toward the average face $(-50 \%)$, and a $25 \%$ caricature away from the average $(+25 \%)$. Participants were asked to choose the most attractive face from a pair that included the veridical face and one of the manipulated faces. The dependent variable was the proportion of trials in which the face that was closer to the average was chosen.

Stimuli. Digital photographs of 16 white females (aged between 18 and 30) in full-face and profile views with neutral expressions were selected. All faces were unfamiliar to the participants. None wore any jewelry or glasses.

The interpupil distance in each full-face image was standardized by scaling the images, and the position of the pupils was matched. The profile views were scaled and aligned vertically to match the position of the top of the head and the underside of the chin. The images were moved horizontally to overlie the position of the facial landmarks. The photographs were manipulated using Morph (Gryphon Software). One hundred twenty landmark points were located by hand on the full-face and the profile view of each face. These points defined the internal features, the shape of the face, and the hair. The software joined subsets of these points to provide a smooth line "drawing" of the face. First, a separate average of the full-face and profile views of the faces was derived. Pairs of photographs were "morphed" successively to produce a single average image, to which all 16 faces had made an equal contribution. Differences in the position of the landmarks were interpolated across pairs of faces. The software automatically fitted the average of the texture of the faces to the shape given by the new landmark positions. Sixteen faces were used to generate the average face views because Langlois and Roggman (1990) found that a composite of 16 faces is judged as more attractive than the individual faces contributing to it.

The average face in the appropriate view was used as a reference image to produce the manipulated full-face and profile images. Each original veridical $(0 \%)$ face from the set was morphed to reduce the differences between the veridical and the average by $25 \%$ to produce the $-25 \%$ image, and by $50 \%$ to produce the $-50 \%$ image. The $+25 \%$ caricature image was created by increasing this difference by $25 \%$. The stimulus set comprised a total of $128 \mathrm{im}-$ ages; one full-face and one profile view at each level of manipulation $(-50 \%,-25 \%, 0 \%$, and $+25 \%)$ for each of the 16 faces. The images were smoothed to reduce the differences in the quality of the image between the veridical and manipulated images, first using the "jaggy despeckle" filter (symmetrical, 3-pixel radius), followed by the Gaussian blur filter (radius 2 pixels) in Corel Photo Paint (Version 8.232). Finally, the images were converted to grayscale.

Procedure. Images were presented on a laptop computer with a 12-in. (304-mm) color liquid crystal screen with a resolution of $800 \times$ 600 pixels. Stimuli were presented using the E-Prime experiment generator (Psychology Software Tools). Each full-face image was $200 \times$ 300 pixels, and each profile view was $170 \times 300$. Images were presented in pairs, one on the left of the screen and one on the right. Each participant was asked to pick which face was the more attractive and responded by pressing a key relating to the left-hand or the right-hand image. The next image was then presented. The comparisons always included the veridical $(0 \%)$ image and one of the manipulated images $(-50 \%,-25 \%$, or $+25 \%)$. The location of images on the left or right was randomized. Each participant judged the faces in either the fullface or profile view and then completed the same comparisons in the alternate view. The stimuli were presented in a different random order for each participant, and the order of views was counterbalanced.

\section{Results}

The proportion of trials in which the more average of the two alternatives was chosen is shown in Table 1. Bi- 
Table 1

Proportion of Trials in Which the More Average Face Was Selected as More Attractive in a Two-Alternative Forced Choice of Female Faces

\begin{tabular}{lccc}
\hline \multicolumn{1}{c}{ View } & Choice & Proportion & Probability \\
\hline Full face & $-50 \%$ vs. $0 \%$ & $93 \%$ & $<.001$ \\
Full face & $-25 \%$ vs. 0\% & $86 \%$ & $<.001$ \\
Full face & $0 \%$ vs. $+25 \%$ & $68 \%$ & $<.001$ \\
Profile & $-50 \%$ vs. $0 \%$ & $76 \%$ & $<.001$ \\
Profile & $-25 \%$ vs. $0 \%$ & $75 \%$ & $<.001$ \\
Profile & $0 \%$ vs. $+25 \%$ & $78 \%$ & $<.001$ \\
\hline
\end{tabular}

Note-The data are shown as a function of the view of the pair of faces shown and the percentage manipulation relative to the average face. $0 \%$ denotes the veridical image; $+25 \%$ denotes $25 \%$ caricature away from the average; $-25 \%$ and $-50 \%$ denote $25 \%$ and $50 \%$ anticaricature toward the average. "Proportion" denotes the mean proportion of trials in which the first manipulation level listed was selected. For all pairs, this is the choice predicted by the averageness hypothesis. "Probability" denotes the statistical probability that the preference differs from chance $(50 \%)$ by a binomial test.

nomial test showed that in all comparisons this proportion differed significantly from chance $(50 \%)$.

The proportion of trials in which the more average face was preferred served as the dependent variable in a repeated measures analysis of variance (ANOVA) with participants as the random factor $\left(F_{1}\right)$. There were two within-participants factors, view and manipulation level. The data were additionally analyzed in an ANOVA with the person photographed as the random factor $\left(F_{2}\right)$. In this items analysis, manipulation level and view formed within-items factors. In both analyses, Mauchly's sphericity tests revealed heterogeneity of covariance. Application of the Greenhouse-Geisser correction did not alter the interpretation in either case, so the unadjusted ANOVAs are presented.

There was a significant effect of view $\left[F_{1}(1,47)=\right.$ $18.11, M S_{\mathrm{e}}=0.248, p<.001 ; F_{2}(1,15)=6.225, M S_{\mathrm{e}}=$ $0.082, p<.05]$ and of manipulation $\left[F_{1}(2,94)=24.56\right.$, $M S_{\mathrm{e}}=0.291, p<.001 ; F_{2}(2,30)=6.154, M S_{\mathrm{e}}=0.101$, $p<.05]$. However, the main effects were qualified by a significant two-way interaction $\left[F_{1}(2,94)=33.41, M S_{\mathrm{e}}=\right.$ $0.462, p<.001 ; F_{2}(2,30)=15.575, M S_{\mathrm{e}}=0.151, p<$ $.001]$.

For the full-face views, the $-50 \%$ and $-25 \%$ anticaricatures were preferred to the $0 \%$ veridical, and the veridical view was preferred to the $+25 \%$ caricature. The same pattern emerged in the profile views. Analysis of simple main effects of the significant interaction showed that the preference for the $50 \%$ and for the $25 \%$ anticaricature was greater in the full-face view than in the profile view $\left[F_{1}(1,47)=45.72, M S_{\mathrm{e}}=0.015, p<.001\right.$; $F_{2}(1,15)=14.29, M S_{\mathrm{e}}=0.015, p<.005 ; F_{1}(1,47)=$ $22.64, M S_{\mathrm{e}}=0.012, p<.001 ; F_{2}(1,15)=7.47, M S_{\mathrm{e}}=$ $0.012, p<.02$, respectively]. The preference for the veridical over the $25 \%$ caricature was stronger in the profile view than in the full-face view $\left[F_{1}(1,47)=15.65\right.$, $M S_{\mathrm{e}}=0.015, p<.001 ; F_{2}(1,15)=14.07, M S_{\mathrm{e}}=0.012$, $p<.02]$.

\section{Discussion}

The preference for full-face views of faces that are closer to the average shape, reported in previous research, was replicated. In all three comparisons tested, participants showed a strong preference for the more average of the stimuli. These results could reflect a preference for either average faces or symmetrical faces or for both factors, since they are confounded in the manipulation of full-face views. The comparison between profile views of faces showed that profile views of faces that were more average in shape were preferred in all three comparisons tested. These data demonstrate a preference for averageness per se in female faces that cannot be attributed to an effect of symmetry. Thus these data provide support for the averageness hypothesis.

The preference for faces that are closer to the average shape was stronger in the full-face views than in the profile views. This difference is consistent with part of the preference for more average full-face views of faces being attributable to increased symmetry. However, the possibility of a contribution from some other aspect of full-face views cannot be excluded. For example, the effect of the image manipulation may simply be more perceptually salient in a full-face view. The greater preference of the veridical over the $25 \%$ caricature in the profile view than in the full-face view does not appear to have any theoretical interpretation. Figure 1 suggests that the $25 \%$ caricature of the profile view produced an extreme shape, especially to the nose, that did not occur in the full-face view.

One effect of the averaging procedure is that the anticaricatured faces tend to have a slightly smoother skin texture. Any blemishes or texture elements from individual faces contribute less to images that are closer to the average face. A caricature will tend make a blemish more prominent. All judgments made in Experiment 1 were between a veridical face and a morphed face (either an anticaricature or a caricature). The images were processed to reduce this artifact; inspection of Figure 1 shows that the differences in texture are slight. Nevertheless, these data cannot exclude the possibility that participants may have been influenced by these slight differences in skin texture.

\section{EXPERIMENT 2}

Two changes were introduced in Experiment 2 to minimize any influence of smoother skin texture in morphed images. First, participants were asked to make a choice between the perceived attractiveness of the $25 \%$ and $50 \%$ anticaricatures. Although the $50 \%$ anticaricature is closer to the average than is the $25 \%$ caricature, this provides a strong test of the effects of averageness since both images are more average than the original. The skin texture of the $50 \%$ anticaricature may still appear slightly smoother (Figure 1). Further processing with a Gaussian filter that blurred the image further reduced this difference (Figure 2). In all other aspects, the images used 


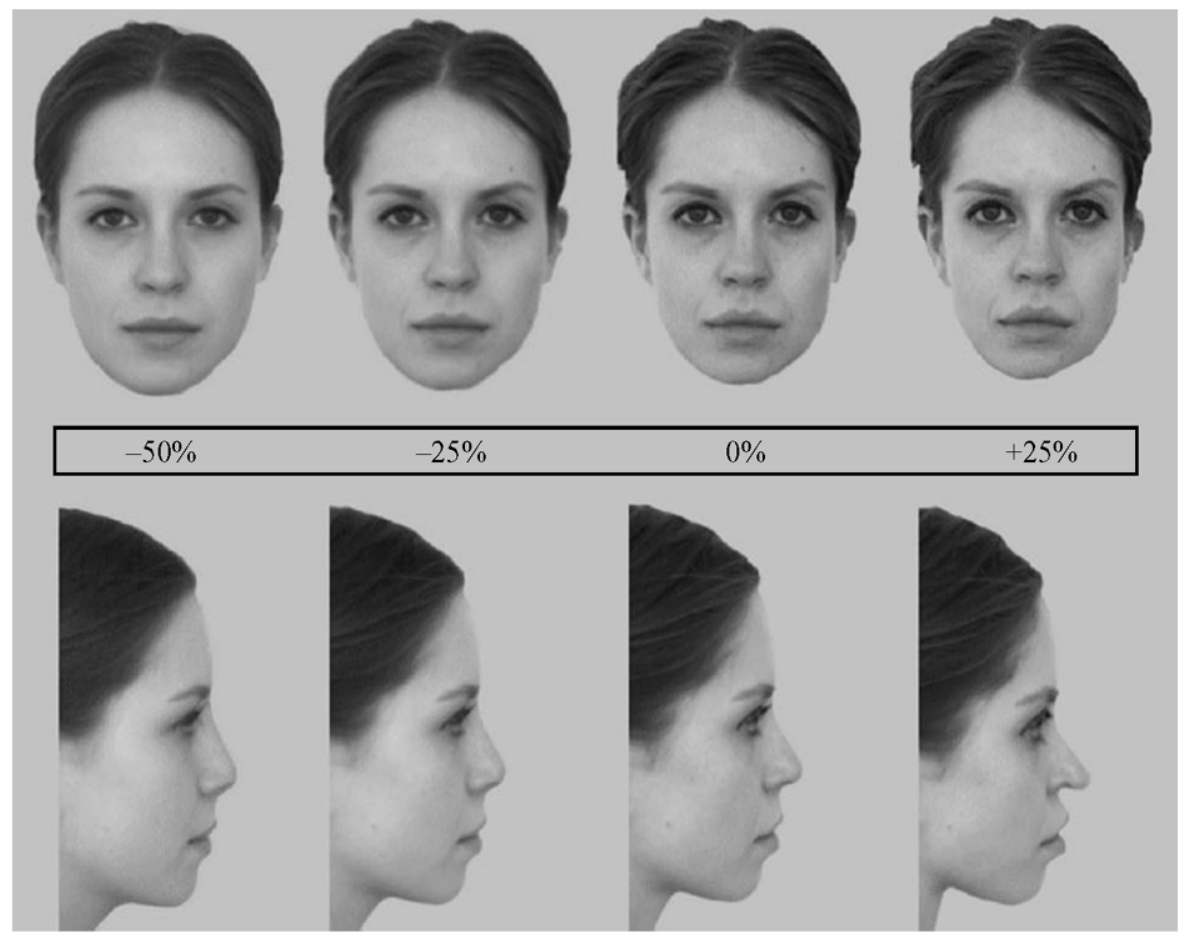

Figure 1. An example of the stimuli. $0 \%$ denotes the veridical image; $+25 \%$ denotes $25 \%$ caricature away from the average; $-25 \%$ and $-50 \%$ denote $25 \%$ and $50 \%$ morph toward the average (anticaricature).

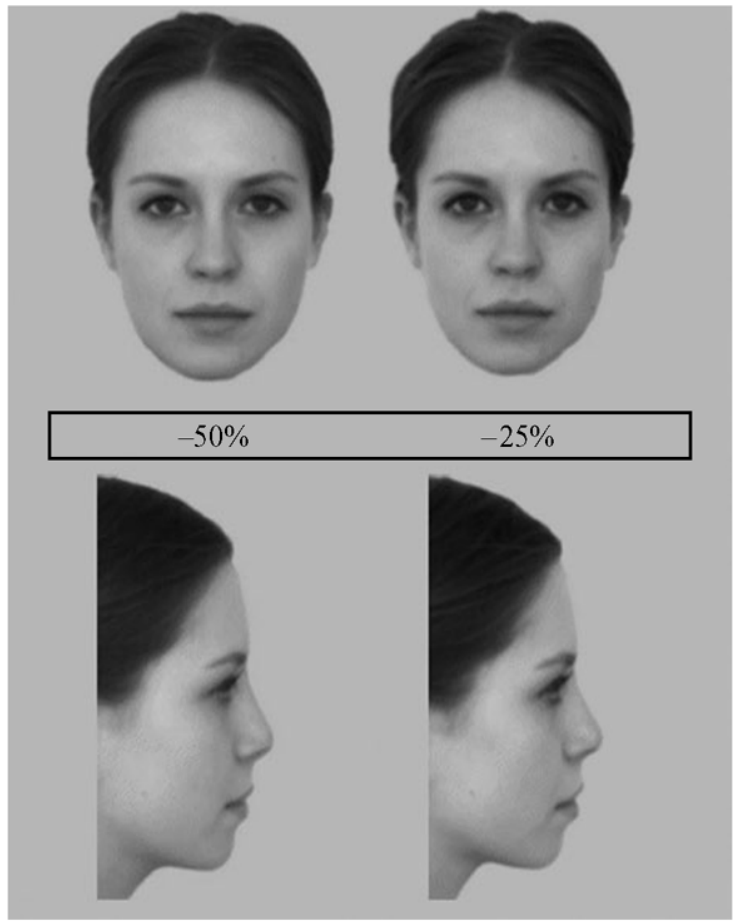

Figure 2. An example of the stimuli used in Experiment 2. $-25 \%$ and $-50 \%$ denote $25 \%$ and $50 \%$ morph toward the average (anticaricature). Note that more blur was applied to the images in Experiment 2 in order to better equate the quality of the images. 
were identical to the $-25 \%$ and $-50 \%$ images used in Experiment 1. The procedure for Experiment 2 was the same as for Experiment 1 except that participants were asked to make only a single choice between two versions of each face. Thus each individual face appeared on only one trial in each view.

\section{Method}

Participants. There were 48 student participants ( 35 female, 13 male). Their ages ranged from 18 to 50 years $(M=23$ years, 10 months; $S D=6$ years, 8 months).

Design. The experimental design had one factor, view of face, which was either full face or profile.

Stimuli and Procedure. The stimuli in Experiment 2 were produced in exactly the same way as those in Experiment 1, with the exception that the images were subjected to further smoothing using the Gaussian blur filter (radius 4 pixels) in Corel Photo Paint. The image pairs presented to participants consisted of the $-25 \%$ image and the $-50 \%$ image of an individual face on all trials. There were 32 trials, one pair of full-face and one pair of profile images for each of the 16 faces in the stimulus set. The location on the left or right of the screen of the $-25 \%$ and $-50 \%$ morphs was randomly assigned on each trial. All full-face trials were presented in one block, and all profile trials in another block. The order of these blocks was counterbalanced.

\section{Results}

The proportion of trials in which the more average of the two images was chosen was $75 \%$ for the full-face views and $67 \%$ for the profile views. Binomial test showed that both proportions differed significantly from the proportion expected by chance $(50 \%, p<.001$, for both tests). A related $t$ test confirmed that the proportion choosing the more average face $(-50 \%)$ was higher for the full-face than for the profile views $[t(47)=2.926$, $p<.01]$. A related $t$ test taking faces rather than participants as the random factor gave a similar result $[t(15)=$ $2.686, p<.02]$.

\section{Discussion}

The $50 \%$ anticaricature was preferred to the $25 \%$ anticaricature in both profile and full-face views. The preference was significantly stronger in the full-face view. The preference of the $50 \%$ anticaricature in the profile view is not confounded by any effect of symmetry. Any differences in the smoothness of the skin texture were substantially reduced by further processing of the images and were substantially less salient than were the differences in shape (Figure 2). Therefore, this significant preference provides good evidence of the influence of averageness per se on the attractiveness of female faces. The preference for the $50 \%$ anticaricature in the full-face view could be attributed to the combined effects of increased averageness and increased symmetry. The significantly stronger preference for a $50 \%$ anticaricature over a $25 \%$ anticaricature in a full-face view than in a profile view could be attributed to the effect of symmetry or possibly some other aspect of the salience of the morphing process in full-face and profile views.

\section{GENERAL DISCUSSION}

In previous research, attempts to separate the influences of symmetry and averageness on facial attractiveness have been made by blending faces to produce symmetrical but not average faces or by using statistical techniques. These approaches suggested an independent role for both factors but could not completely isolate the influence of each factor. The two present experiments examined the perception of attractiveness in profile views of faces, allowing the influence of averageness to be examined in the total absence of any influence of symmetry. Both experiments showed that the attractiveness of a profile view of female faces is influenced by the averageness of the profile. Also, both experiments showed that the influence of making a face more average in appearance was significantly stronger in a full-face view than it was in a profile view. The latter result is consistent with an independent influence of symmetry on attractiveness of full-face views, but could be attributed to some other aspect of full-face views. Artifacts due to the smoothing of skin texture were substantially reduced in Experiment 2, but the same pattern of results was observed as for Experiment 1. Taken together with the results of previous work, there is considerable converging evidence from a range of methods that both averageness and symmetry have independent effects on the perception of facial attractiveness.

Halberstadt and Rhodes $(2000,2003)$ found that the preference for average stimuli is not limited to human faces; perceived attractiveness and averageness are correlated for images of dogs, birds, watches, fish, and automobiles. These results suggest that a preference for the average of a familiar category may be a consequence of general cognitive processing. It could be argued that evolution has selected for a cognitive system that provides a preference for average or symmetrical human faces because of an adaptive advantage (i.e., the "good genes" account). The preference for the average of other categories may be simply a redundant, emergent property. Alternatively it could be argued that the preference for average faces is an emergent property of our object and face recognition systems and serves no adaptive function (i.e., a perceptual bias account).

A critical requirement for distinguishing between a good genes account and a perceptual bias account is to establish whether facial attractiveness does honestly signal health. There is only weak evidence to support such a relationship (Langlois et al., 2000). The most comprehensive study available showed that facial attractiveness in late adolescence is not associated with either current or future health (Kalick et al., 1998). Indeed, perceived attractiveness acted to suppress the relationship between perceived health and medical assessments of health. In contrast, Rhodes, Zebrowitz, et al. (2001) found a modest negative correlation between facial distinctiveness and current health for female faces and between facial 
distinctiveness and childhood health for male faces. As distinctive faces differ from the average face more than typical faces, these results suggest an association between averageness and health. However, there is no a priori rationale for the difference in the associations found for male and female faces. No relationship between health and facial symmetry was found for either male or female faces. The present balance of evidence appears to favor a perceptual bias account of human preferences for average and symmetrical faces.

\section{REFERENCES}

Galton, F. (1878). Composite portraits. Journal of the Anthropological Institute of Great Britain \& Ireland, 8, 132-144.

Halberstadt, J., \& Rhodes, G. (2000). The attractiveness of non-face averages: Implications for an evolutionary explanation of the attractiveness of average faces. Psychological Science, 11, 285-289.

Halberstadt, J., \& Rhodes, G. (2003). It's not just average faces that are attractive: Computer-manipulated averageness makes birds, fish, and automobiles attractive. Psychonomic Bulletin \& Review, 10, 149156.

Kalick, S. M., Zebrowitz, L. A., Langlois, J. H., \& Johnson, R. M. (1998). Does human facial attractiveness honestly advertise health? Longitudinal data on an evolutionary question. Psychological Science, 9, 8-13.

KowNER, R. (1996). Facial asymmetry and attractiveness judgment in developmental perspective. Journal of Experimental Psychology: Human Perception \& Performance, 22, 662-675.

Langlois, J., KalaKanis, L., Rubenstein, A. J., Larson, A., HalLAM, M., \& SмоOт, M. (2000). Maxims or myths of beauty? A metaanalytic and theoretical review. Psychological Bulletin, 126, 390-423.

Langlois, J., \& Roggman, L. A. (1990). Attractive faces are only average. Psychological Science, 1, 115-121.

Langlois, J., Roggman, L. A., \& Musselman, L. (1994). What is average and what is not average about attractive faces? Psychological Science, 5, 214-220.

LEE, K. J., $\&$ PERRETT, D. I. (2000). Manipulation of color and shape information and its consequence upon recognition and best-likeness judgments. Perception, 29, 1291-1312.

Perrett, D. I., Burt, D. M., Penton-VoAK, I. S., Lee, K. J., Rowland, D. A., \& EDwARDs, R. (1999). Symmetry and human facial attractiveness. Evolution \& Human Behavior, 20, 295-307.

Perrett, D. I., Lee, K. J., Penton-Voak, I. [S.], Rowland, D., YoshiKawa, S., Burt, D. M., Henzi, S. P., Castles, D. L., \& Akamatsu, S. (1998). Effects of sexual dimorphism on facial attractiveness. Nature, 394, 884-887.
Perrett, D. I., May, K. A., \& Yoshikawa, S. (1994). Facial shape and judgements of female attractiveness. Nature, 368, 239-242.

RHODES, G. (1996). Superportraits: Caricatures and recognition. Hove, U.K.: Psychology Press.

Rhodes, G., Profitt, F., Grady, J. M., \& Sumich, A. (1998). Facial symmetry and the perception of beauty. Psychonomic Bulletin \& Review, 5, 659-669.

RHODES, G., Roberts, J., \& Simmons, L. W. (1999). Reflections on symmetry and attractiveness. Psychology, Evolution, \& Gender, $\underline{1}_{2}$ 279-295.

RhODEs, G., Sumich, A., \& ByatT, G. (1999). Are average facial configurations attractive only because of their symmetry? Psychological Science, 10, 52-58.

RHODES, G., \& TREMEWAN, T. (1996). Averageness, exaggeration and facial attractiveness. Psychological Science, 7, 105-110.

Rhodes, G., Yoshikawa, S., Clark, A., LeE, K., McKay, R., \& AKaMATSU, S. (2001). Attractiveness of facial averageness and symmetry in non-Western cultures: In search of biologically based standards of beauty. Perception, 30, 611-625.

Rhodes, G., Zebrowitz, L., Clark, A., Kalick, S. M., Hightower, A., \& McKAY, R. (2001). Do facial averageness and symmetry signal health? Evolution \& Human Behavior, 22, 31-46.

Samuels, C. A., Butterworth, G., RoBerts, T., Graupner, L., \& Hole, G. (1994). Facial aesthetics: Babies prefer attractiveness to symmetry. Perception, 23, 823-831.

Thornhill, R., \& GANGESTAD, S. W. (1999). Facial attractiveness. Trends in Cognitive Science, 3, 452-460.

VALENTINE, T. (1991). A unified account of the effects of distinctiveness, inversion and race in face recognition. Quarterly Journal of Experimental Psychology, 43A, 161-204.

VALENTINE, T. (2001). Face-space models of face recognition. In M. J. Wenger \& J. T. Townsend (Eds.), Computational, geometric, and process perspectives on facial cognition: Contexts and challenges (pp. 83113). Mahwah, NJ: Erlbaum.

\section{NOTE}

1. There may be some aspects of a face, perhaps those associated with sexual dimorphism, for which there is directional selection for extreme rather than average values. For example, participants prefer feminized female and male faces over average faces (Perrett et al., 1998). This hypothesis is analogous to a directional selection preference for a long peacock's tail (see Rhodes, 1996, for a discussion). Selection for extreme attributes cannot provide an account for the perception of average or symmetrical faces as attractive, and therefore was not relevant to the issue considered here.

(Manuscript received February 24, 2003; revision accepted for publication June 18, 2003.) 